\title{
Mental Health and Self-help Method
}

\author{
Jumala Yogitha
}

yogithajumala@gmail.com

\begin{abstract}
In the 21st century, any pandemic, especially, SARS-CoV-2 is a global burden due to high incidence, mortality, and mutation rate. Although several techniques have already been identified to control the pandemic or treat patients and causes of adverse impact on mental health, relatively only, fewer researchers have little concern about finding effective mitigation strategies to improve mental health. Therefore, this study aimed to find some common and unique approaches to manage mental health during a pandemic. Some strategies for the better management of mental health induced by SARS-CoV-2 infections are required for all classes of peoples. Early management is vital, and those must be associated with frontline workers and people staying at home, particularly in isolation centers and already identified as active cases. Experts have pointed out the need to pay specific attention to proper daily life. To manage abnormal mental conditions, such as anxiety, mood, personality, and psychotic disorder during the pandemic; social media, meditation, and psychological motivation with adequate diet, exercise, and sleep have significant roles in regulating some biological mechanism, incredibly immune, hormonal, and neural process. Management of mental health is mandatory for all at the time of the SARS-CoV-2 pandemic. We can consider all of the strategies mentioned above to treat mental health during and after the COVID- 19 pandemic condition.
\end{abstract}

\section{Introduction}

The outbreak of SARS-CoV-2 is considered both epidemic and pandemic in the world. Currently, people all over the world have been affected by SARS CoV-2, which is the fifth pandemic after the 1918 flu pandemic [1]. COVID-19 is turning into COVID-21 by crossing COVID-20, but infections and death are increasing with generating several problems. The outbreak caused by SARS-CoV-2 likely to cause an increase in global issues like economic burden, poor healthcare infrastructure [2], social issues, [3]political problems, hunger or malnourishment, and lastly, the most magnitude is the mental health problem in both developed and developing countries [4, 5].Accumulating evidence suggests that SARS-CoV-2 mediated outbreak can damage psychological or mental health during a pandemic which is not something new as people have faced a similar crisis in the past year for every pandemic like the Ebola virus disease outbreak [6], SARS, MERS [7] influenza outbreak $[8,9]$. Longtime epidemics can negatively impact people in several ways, such as feelings of personal danger of infection, concern for relatives as well as family [10]. Furthermore, losing jobs, decreased earnings, unpayable loans due to low income are indicative causes of mental stress [11].

Shammi (2020) reported that longtime lockdown leads to unemployment, demotion, and hunger problem due to the food shortage of poor people that ultimately contribute to panic and mental stress in people [12]. Zhai and Du (2020) conclude that inadequate healthcare facilities in some countries, i.e., limited testing that is also confined to the urban area and inadequate treatment facilities, negatively impact public mental health [13]. A recent study by Magson et al. summarized that COVID-19 related worries, teething troubles of learning online, and raised family disagreement were also associated with the greater psychological problem [14]. Widespread rumors and misinformation in social media and indirect exposure to continuous news may have had a wide variety of psychological effects, including stress-related disorders [15]. Moreover, some additional factors, including inadequate material supplies, businesses failure, travel shut down, mutation of the virus, and conflicting vaccination, may also cause psychological problems. As a consequence, these factors may trigger feelings of anger, anxiety, grief, loneliness, boredom and may lead to severe mental health problem $[16,17]$.

Moreover, this mental health crisis may also increase other problems like xenophobia, suicide, heart problem, insomnia, stroke, chronic pain, threatening behavior, erratic or unusual behavior, self-injury, paranoid thinking, and drug addiction among the people [18, 19]. The previous studies conclude that pandemic induced mental problems result in post traumatic stress disorder (PTSD) symptoms, including nightmares, hyperarousal, detachment, numbing, and the risk of infections such as pneumonia [20, 21]. The metaAnalysis by Pappa et al. amalgamated thirteen studies with a total of 33,062 participants and reported that 12 reviews were anxiety positive which is accounted for $23.2 \%$, whereas depression was consistent in 10 studies, with an incidence rate of $22.85 \%$ [22]. Insomnia was another prominent mental health issue with having an incidence rate of $38.9 \%$ per 5 reports by the same author. Furthermore, descriptive analyzes have been performed and found that $18.7 \%$ of the sample revealed depressive behavior, $21.6 \%$ anxiety, and $15.8 \%$ PTSD symptoms [23]. Data about mental health status caused by SARS-CoV-2 is available globally, but the proposed mechanism for managing this mental health-related problem is rare. It may be due to lack of available information. Therefore, in this review, the authors proposed few possible strategies to manage mental health during COVID-19 pandemic.

\section{Management of Mental Health during COVID -19 Pandemic}

Psychological distress, i.e., insomnia, alcohol/drug misuse, and symptoms of posttraumatic stress disorder (PTSD), depression, anxiety, burnout, anger, and higher perceived stress, are normal during disasters like the COVID-19 pandemic. People, particularly doctors as well as other health care practitioners (hospital staff members, receptionists, cleaners, and caterers), are susceptible to the adverse effects of psychological disorders because they seek to balance the responsibility of caring for patients, 
their families, and friends. It's essential to improve people's mental and physical well-being to minimize distress and slow the occurrence of serious problems such as depression or anxiety. There are good evidence-based guidelines in this section that can reduce the negative impact and promote well-being. Following are the possible ways to manage mental health during this pandemic.

\section{Exercise}

The COVID-19 pandemic made people fixed at home (isolation and quarantine), and physical and social activity was decreased which led to increased emotional or behavioral disorders like fear, terror, depression, anxiety. The results from previous performed observational and experimental studies demonstrated that regular physical exercise could suggestively progress mental health and reduce depression, nervousness, and stress symptoms [2429].

In recent research on physical activity, physical exercise is recommended for both indoors and outdoors people during the COVID-19 outbreak as it is beneficial for boosting up the immune system to relieve anxiety and depression [2932]. Studies have shown that several types of exercise effectively lower blood pressure, enhance cardiovascular health, and prevent various cancers, as well as diabetes, osteoporosis, hypertension, obesity, and Alzheimer's disease [25-28]. Studies on animal models have also shown that exercise positively affects depression by controlling neurotransmitters, neurogenesis, neurotrophic factors, and cerebral blood flow. In vivoexperiment conducted by Sleiman SF et, al. reported that exercise is a factor that appears to raise the amount of brain-derived neurotrophic factor (BDNF) in the hippocampus, which is shown to combat sadness and anxiety in mice by storing an endogenous molecule, D- $\beta$-hydroxybutyrate (DBHB). Besides, regular physical activity will reduce BDNF levels and provide neuroprotection and neurotrophic impact in a similar model [33]. A study from Gokdemir et al. and Daniele et al. reported that exercise could help increase norepinephrine (NE) levels and serotonin metabolites in the mouse brain to prevent depressive behavior and neurochemical shift. [34, 35].

Long-term research carried out by Wu et al. on TgF344-AD transgenic, and wild rats concluded that exercise reduced anxious-depressed behaviors and enhanced fear-preventing action by controlling amyloid- $\beta$-deposition, hyperphosphorylation, microgliosis, inflammatory cytokines release, and oxidative damage [24]. A clinical study in twenty-one participants by Passos et al. summarized that long term moderate aerobic exercise training improved sleep, lowered depression and cortisol, and enhanced significant changes in immunologic variables like increased plasma apolipoprotein A and decreased CD4 and CD8 [36]. In another study, Woods et al. summed up that routine exercise protects against neural diseases such as dementia and depression through potential pathways including weight loss, declines in macrophage accumulation in adipose tissue, macrophage modifications, exerciseinduced muscle development of IL-6, for the effect of anti inflammatory activities [37]. Exercise is compared to antidepressant medication as a first-line treatment for mild to moderate depression. For instance, animal models show that daily aerobic activity improves brain serotonergic and noradrenergic levels similar to antidepressants effects. [38] Anxiety is the most prevalent type of mental illness, causing disturbed sleep, mood changes, and difficulty completing ordinary tasks [39]. Vogelzangs and Michopoulos reported that exercise could regulate proinflammatory cytokines and C-reactive protein (CRP) via the inflammatory response, which improves anxiety symptoms [40]. A study conducted by China on college students showed that regular physical activity could alleviate stress and anxiety induced by COVID-19 or improve mental health behavior [5].

Adlard et al. reported that physical activity was found to accumulate BDNF at pre-stress levels, meaning that the exercise protects from stress-related lower BDNF levels [41]. Furthermore, a recent study stated that relaxation and breathing strategies were recommended as potential measures to improve sleep quality, anxiety, and depression in COVID-19 patients

[42].

According to Liu et al., the anxiolytic effect is due to enhancing hippocampal neurogenesis and restoring neurotransmission of neuropeptide Y (NPY), while Lopresti et al. described in his literature that exercise helped to upregulate hypothalamic-pituitary-adrenal (HPA) axis in animal- and human-based studies [43, 44]. Exercise can help to minimize inflammation and lead to improved mental health outcomes for patients with inflammatory disorders. For instance, a study in eighty patients by Abd El-Kader et al. showed that exercise might minimize inflammation through reducing necrosis factor-alpha (TNF- $\alpha$ ), interleukin-4 (IL-4), interleukin-6 (IL-6), and C-reactive protein (CRP) [45]. In a meta-analysis study by Heyn et al., exercise results in older people with cognitive disability and dementia showed that practice instruction increases fitness, physical performance, cognitive function, and constructive actions. [46] Keeping mentally and physically active during the COVID-19 outbreak will help reduce the negative impact on mental health following the COVID- plague. As a whole, further investigation is needed to prove the efficiency of exercise as a first-line or active treatment. But we all can agree that exercise is an effective way of decreasing stress, depression, and anxiety based on the data and previous research. Overall, exercise can be a therapeutic way to manage mental health-related disorders during an emergency situation, depicted in Figure 1.

\section{Diet}

A proper diet during the COVID-19 pandemic is vital. Although no food or nutritional supplements can prevent infection with SARS-CoV-2, good diets are essential to promote immune systems and improve psychological conduct. According to the World Health Organization (WHO), eating healthy, frozen, and unrefined foods every day to get the body's needs in terms of vitamins, minerals, dietary fiber, protein, and antioxidants is also a safe way with better immune systems and a minor risk of chronic conditions and infectious illnesses [47]. This section provides scientific information and evidence on the 
importance of food and bioactive ingredients in health management.

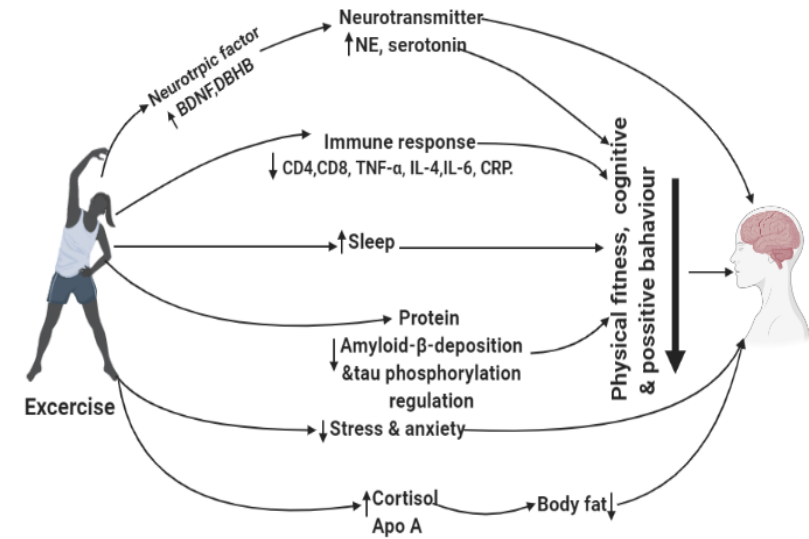

Figure 1. Possible mechanistic way of managing mental health by exercise during pandemic. Neural development is possible by regular and proper exercise through upregulating BDNF, DBHB, neurotransmitter and regulating neurodegenerative diseases related to protein aggregation. Furthermore, exercise enhances immune response, sleep, and hormone-related brain functions, leading to improved physical fitness and cognitive and positive behaviors. BDNF-Brain-derived neurotrophic factor; DBHB- $\beta$-hydroxybutyrate; NE-Norepinephrine; CD4 \&8-Cluster of differentiation $4 \& 8$; TNF- $\alpha$ - Tumor necrosis factor alpha; IL-4 \& 6-Interleukins-4\&6; CRP- C reactive protein; Apo A- Apolipoprotein A.

\section{Carbohydrate}

[49]. Rodríguez-Valentín et al. reported in their in vitro study that mushroom polysaccharides were associated with anti-HIV activity through suppressed virus replication and upregulation of some antiviral chemokines, including macrophage inflammatory protein (MIP-1 $\alpha / \beta)$ and stromal cell-derived factor-1 alpha $(\mathrm{SDF}-1 \alpha)$ [50]. Some carbohydrates significantly impact memory development during short, stressful times by releasing cortisol [51]. Several studies have shown that carbohydrate consumption enhances the ability to focus, response speed, processing ability, mood, and memory, while disturbances in carbohydrates raise the risk of cognitive memory disorders [52]. Randomized Controlled Trial by Owen et al. suggests that higher carbohydrate doses can affect memory in both hippocampal and non hippocampal brain regions [53]. A systematic review conducted by Hoyland (2009) summarized that eating a regular breakfast or consuming glucose can help enhance mental efficiency by increasing memory effectiveness with attention task capability than no breakfast group [54]. Clinical data suggest that a low glycaemic index (GI) breakfast allows healthier cognitive activities in rats and humans [55].

\section{Protein}

Dietary protein and individual amino acids like tryptophan and tyrosine consumption help improve cognitive development and brain function discussed in observational and interventional studies by van de Rest (2013) [56]. Highquality protein foods (meats, milk, and others, eggs) improve the brain's functioning and mental health by producing neurotransmitters like dopamine and serotonin from amino acid tyrosine and tryptophan [48]. An experimental study conducted by Khaliq et al. reported that a high dose of tryptophan $(50-100 \mathrm{mg} / \mathrm{kg}$ ) and serotonin or their metabolites improve cognitive performance in the bred albino Wister rats (150-160 g) model [57]. Based on a previous study, it can be suggested that foods rich in protein can help manage mental health induced by the SARS-CoV2 infection; also, WHO suggests that consuming protein from animal sources, including fish, meat, milk, and eggs as high calorie and high protein diets are considered necessary to prevent or attenuate the loss of lean muscle in COVID-19 patients [58]. In a study, Muscogiuri et al. highlighted that protein-rich foods such as milk, yoghurt, seeds, and nuts could be a better source of tryptophan (an amino acid and serotonin precursor) during the quarantine period of COVID-19 to manage mental health [59].

\section{Essential fatty acids}

The fundamental role of fat in immunity has been established in the literature; for instance, monounsaturated and polyunsaturated fatty acids have a beneficial immune modulated impact. [60]. These bioactive lipids also exhibit macrophage phagocytic ability and have a beneficial effect in 280 treating a viral infection [61]. There are a lot of essential fatty acids, like omega- 6 and omega-3, may be effective in preventing and controlling SARS-CoV-2 infection and other enveloped viruses with inhibiting angiotensin-converting enzymes (ACE, an ACE-2 precursor), decreasing the supply of SARS-CoV-2 receptors and subsequently making unable to reach the target cell [62, 63]. Furthermore, in vivo studies in the patients suspected of being COVID- 19 showed that lipids including fatty acids, phytosterols, and carotenoids improve immune response, antiinflammatory activity, decrease the risk of cardiovascular disease by inhibiting ACE 4 receptors as well as limiting virus ability to reach cells [64, 65]. The omega-3 fatty acids, especially alpha-linolenic acid (ALA), are essential for the brain's structure and function. It's been assumed that sufficient polyunsaturated fatty acids (PUFAs), especially Docosahexaenoic acid (DHA), inhibit the development of depression [48].

\section{Vitamin}

Vitamin is a crucial participant in the army of immunity. Carr et al. conclude that vitamin supplements can benefit those vulnerable to respiratory virus infections; for example, vitamin $\mathrm{C}$ is a potent source of antioxidants and caused reactive oxygen species (ROS) mediated phagocytosis and chemo taxis to destroy the viral pathogen [66]. The main comorbidities of COVID-19 patients are common cold, diabetics, cardiovascular, kidney, cancer, the microbial infection can be prevented with the help of consuming antioxidant vitamin $\mathrm{C}$ through promoting immune functions, autophagy inducing, and reducing inflammation and oxidative stress by lowering NF-kB, CRP, and ILs [67]. McCartney et al. reported that vitamin $\mathrm{D}$ shortage is thought to down regulate cluster of differentiation 2 (CD26), interleukin-6 (IL-6), and interferon-gamma (IFN $\gamma$ ) those are considered molecule for COVID-19 host cell defense system [68].

Baksi et al. confirmed that severe vitamin D shortage in young rats could elevate catecholamine levels in the brain [69]. Furthermore, a study conducted by Benton et al., 
supplementation of nine B-complex vitamins especially, vitamin B2 and B6 status, ten times over standard recommended dietary allowance (RDA) for the 1-year improved mood in both men and women [70]. Clinical studies have shown that vitamin B12 prevents the onset of dementia symptoms. The addition of cobalamin stimulates older people's brain and cognitive functions; It also supports frontal lobe factors [71]. During the COVID-19 pandemic, sufficient zinc and vitamin $C$ and $D$ intake may be a promising pharmacological tool for preventing starting the inflammatory process [72].

Minerals are essential micronutrients used in DNA synthesis and cell proliferation, regulating both innate and adaptive immune systems like the production of immune cells [73]. Velthuis et al. reported that increasing the intracellular zinc with pyrithione can be a potential inhibitor for RNA viruses replication as well as SARS coronavirus (SARS-CoV) at low concentrations [74]. Furthermore, Golub et al., summarized from some of the experimental animal (rodents, monkeys, human) study, have shown clearly that Zinc deficiency, mainly at the time of pregnancy, leading the loss of neurons and reduced brain volume Furthermore, it can help $\mathrm{T}$ cell proliferation, antibody production and maintain immunity [78, 79]. The iodine helps to energy metabolism in the cerebral cell, and iodine deficiency at the time of pregnancy induces severe cerebral malfunction resulted in cretinism [80]

\section{Dietary phytochemicals}

Phytochemicals use anti-SARS agents due to the ability to inhibit several mechanisms either at the viral entry point or the replication stages or via immunomodulation potentials [81]. Honey is a significant phytochemicals source, and its mechanisms of anti-viral properties and its very vast and unknown [82]. Abedi et al. summarized that honey and its main components inhibit the entry of the virus into the host cell and its replication and modulate the inflammatory cascade [83]. Islam et al. summarized in their literature review that several phytochemicals from $N$. sativa could be an alternative therapy to combat SARS CoV-2 infection [84] through induction of IL-8 [85], increasing CD4+ $\mathrm{T}$ and IFN- $\gamma$ number with activities [86]. A recent clinical study with 200 patients showed that Nigella sativa administration recovered patients

\section{Sleep}

Psychological distress and signs of mental illness are associated with severe outbreaks of COVID-19 infections [90]. Scientists, on the whole, are trying to manage mental health-related disorders caused by COVID-19, where proper sleep can be a significant candidate. Recent studies outlined by Franceschini et al. (2020) suggest that central Italian people who died due to COVID-19 dramatically altered sleep-wake patterns marked by usual early or delayed bedtime average faster waking time decreased naps in the afternoon [91]. According to the world health organization (WHO), they recommended that people in isolation take regular sleep routines to manage mental health [92]. Melatonin is a pineal hormone that responds to darkness during sleep time, and recent studies outlined by Zhang et al. (2020) suggest that melatonin supports critical care patients in the COVID-19 pandemic by reducing anxiety and improving sleep quality [94, 95]. S [96, 97]. stores and relieve cellular stress [100].

Sleep has a direct impact on the maintenance of immunity and the immunological response linked to the psychological problems imposed by COVID-19.

Milrad et al. demonstrate that pro-inflammatory molecules, including interleukin (IL-11, IL-6), TNF- $\alpha$, and CRP, raised due to poor sleep in people, and a higher level of proinflammatory cytokines causes called "cytokine storm" related to mortality in COVID-19 patients [93, 94]. estimated that the night sleep period correlated with the lowest risk of depression/anxiety for teenagers aged 7-12. For men, the lowest depression/anxiety may be related to sleeping $8.5 \mathrm{~h}$ or more, while for females, the period was reported at $7.5 \mathrm{~h}$ or more [108].

An observational study with a total of 2,631 participants by Supartini et al. demonstrated that depressive symptoms and eventually suicidal ideation are linked with low sleep quality, while enhancing the standard of sleep will avoid the occurrence of depression and decrease the risk of suicide [109].

\section{Motivations}

Motivation is the psychological counselling that encourages someone to work on a problem; those are the direct link to stress and physical health while enlightening individuals' activity in a work context [102-105]. So motivations can be an alternative therapy and may become an adjunctive for the management and treatment of the mental health of COVID-19 infected patients by SARS-CoV-2. Motivation is essential for the quarantine people, possible symptoms of suspected case and positive case so that these people keep feet in mentally during the pandemic. The best motivation during a pandemic is that people believe that maintaining quarantine and social distance interventions, including home-stay, have been introduced worldwide to minimize virus transmission [114]. The WHO recommended that motivation from knowing about fact and clear evidence about reducing the risk of infection is needed to manage mental health. [92]. Self-confidence is an immunemodifying psychotherapeutic intervention that can potentially benefit COVID-19 patients. Hannan et al. summarized that improving self confidence through multiple ways like social supports, psychological interventions, food habits, spiritual connections, health supports, and positive thinking can potentially suppress psychological stress, thereby consolidating immune functions. Furthermore, they also report that self-motivation can help improve non communicable diseases such as neurodegenerative and inflammatory bowel diseases. Consequently, self confidence enables an individual to overcome COVID- 19 and reduces the risk of contracting the disease [93].

Firth et al. summarized in their review, professional support has the capability to tackle psychological barriers by improving physical activity [115]. Motivation can help change our thinking, feeling, and behaving, whereas lack of motivation suppresses functioning and well-being. [116].[117]. A well-motivated person can help others like 
suspected to consume a balanced diet, proper sleep, and regularly perform a physical exercise; those will help to mitigate the mental health related problems during the pandemic crisis.

Although the higher incidence rate in the COVID-19 compared to the previous pandemic, the case fertility rate is rare with a higher recovery rate. So, it should not be panic and worried about the ongoing pandemics.

\section{Social media}

SARS-CoV-2 infections turned the world into uncertainty, and the continuous news from social media about the severity of coronavirus infection and mortality rate made people in a puzzle. This kind of information negatively impacts people's mental health [20, 110, 111]. According to some research, social media can be a definite cause of depression. For instance, I. Pantic (2014) summarized that prolonged use of social networking sites (SNS), such as Facebook, may be related to signs and symptoms of depression [14]. But being alarmed about the COVID-19 related news is understandable; individuals can handle current mental health issues, such as limiting the information and being concerned about what one has read, listening to, and seeing. Anyone may feel 283 nervous or upset by a nearly continuous influx of news stories concerning an epidemic. During some hours of the day, check out health providers for detailed alerts and realistic advice to avoid sharing infection-related rumors responsible for nervousness. According to the World Health Organization (WHO), social networks protect mental health during the coronavirus epidemic, but mental health protection depends on personal behavior. [92]. Some news about spraying disinfectants from social media causes panic in general people [120], thus, it is essential to get actual information from authentic sources to manage the mental health-related problem. In COVID-19 patients, social networking offers an interchange of encouragement and makes individuals feel less depressed because they can contact friends and family and feel community support. These will prevent one from boredom, loneliness, and monotonous situations. Social media gives information within a short time rather than moving outside, avoiding the speeding of SARS-CoV-2 infection. Viewers report to feel chills, excitement, and thrills; television is essential to compensate for the painful day-to-day routine during a pandemic situation. Positive newspaper coverage of COVID-19 related news help to prevent mental illness [121].

Ahmad (2020) suggests that authentic social media consumers on what constitutes good and reliable can play an essential role through younger since they use social media information to distribute it to their families and friends [118]. So in the case of the COVID-19 pandemic, educational facilities would be perfect locations to design classes and symposiums that will help students and teachers search, identify and analyze clinical knowledge that can mitigate mental health.

\section{Meditation}

Meditation techniques are being used to treat and avoid a variety of mental disorders as it is a known and common method for controlling stress and mental health with wellbeing and change in both cognitive and memory functions, regulate social and emotional behavior and help to improve in various, cardiovascular, neurological, autoimmune pathologies [114, 115].

\section{Conclusions}

The COVID-19 pandemic is widely regarded as one of the most severe threats to global health systems in the twentyfirst century. The latest coronavirus has developed immense psychological challenges beyond the physical and geographical problem in different subpopulations around every country, especially industrialized countries. Several factors can be attributed to deal with the negative effect of mental health during the disease outbreak. This narrative research discovered that individuals' characteristics might significantly mitigate psychological behavior, including depression, panic attack, stress, emotional disturbance and somatization, suicidal behavior, sleep disorders, anxiety, post-traumatic stress disorder (PTSD) symptoms, and many additional mental health issues. Physical activity and the maintenance of safe sleeping habits may be beneficial. These are considered mitigation techniques for average citizens forced to remain at home, both cost-effective and realistic.

To decrease quarantine-related situational stress, immunosupportive nutrients play an essential function in improving the brain's negative effect. Furthermore, during the time of the pandemic, motivation from a family member as well as others can improve confidence level and support to the management of the psychological problem, especially health workers. Social media can play another vital role in the teenager by publishing authentic news that ill mitigate depression and anxiety in individuals. In COVID-19 patients, meditation works to mitigate psychological behavior by enhancing resilience and improving well-being during an emergency crisis. Overall, our study's knowledge to be valuable, meaningful, and appropriate to cope with mental stress caused by SARS-CoV-2. We recommend all people follow the complete package of guidance, including exercise, diet, proper sleep, motivation, social media, and meditation, to manage mental health during this COVID-19 crisis

\section{References}

[1] Liu YC, Kuo RL, Shih SR. COVID-19: The first documented coronavirus pandemic in history. Biomed J 2020; 43:328-33. https://doi.org/10.1016/j.bj.2020.04.007.

[2] Zheng J. SARS-coV-2: An emerging coronavirus that causes a global threat. Int $J$ Biol Sci 2020;16:1678-85. https://doi.org/10.7150/ijbs.45053.

[3] Sotgiu G, Dobler CC. Social stigma in the time of coronavirus disease 2019. Eur Respir J 2020;56. https://doi.org/10.1183/13993003.02461-2020.

[4] Talevi D, Socci V, Carai M, Carnaghi G, Faleri S, Trebbi E, et al. Mental health outcomes of the covid19 pandemic. Riv Psichiatr 2020;55:137-44. https://doi.org/10.1708/3382.33569. 
[5] Zhang Y, Zhang H, Ma X, Di Q. Mental health problems during the COVID-19 pandemics and the mitigation effects of exercise: A longitudinal study of college students in China. Int $\mathrm{J}$ Environ Res Public Health 2020; https://doi.org/10.3390/ijerph17103722.

[6] Shultz JM, Cooper JL, Baingana F, Oquendo MA, Espinel Z, Althouse BM, et al. The Role of FearRelated Behaviors in the 2013-2016 West Africa Ebola Virus Disease Outbreak. Curr Psychiatry Rep 2016;18:104. https://doi.org/10.1007/s11920-0160741-y.

[7] De Brier N, Stroobants S, Vandekerckhove P, De Buck E. Factors affecting mental health of health care workers during coronavirus disease outbreaks (SARS, MERS \& COVID-19): A rapid systematic review. PLoS One 2020; 15:e0244052. https://doi.org/10.1371/journal.pone.0244052.

[8] Pfefferbaum B, Schonfeld D, Flynn BW, Norwood AE, Dodgen D, Kaul RE, et al. The H1N1 crisis: A case study of the integration of mental and behavioral health in public health crises. Disaster Med Public Health Prep 2012;6:67-71. https://doi.org/10.1001/dmp.2012.2.

[9] Khan S, Siddique R, Xiaoyan W, Zhang R, Nabi G, Sohail Afzal M, et al. Mental health consequences of infections by coronaviruses including severe acute respiratory syndrome coronavirus 2 (SARS-CoV-2). Brain Behav 2021;11:e01901. https://doi.org/10.1002/brb3.1901.

[10] Li S, Wang Y, Xue J, Zhao N, Zhu T. The impact of covid-19 epidemic declaration on psychological consequences: A study on active weibo users. Int $\mathbf{J}$ Environ Res Public Health 2020;17. https://doi.org/10.3390/ijerph17062032.

[11] Frasquilho D, Matos MG, Salonna F, Guerreiro D, Storti CC, Gaspar T, et al. Mental health outcomes in times of economic recession: A systematic literature review Health behavior, health promotion and society. BMC Public Health 2016;16. https://doi.org/10.1186/s12889-016-2720-y.

[12] Shammi M, Bodrud-Doza M, Towfiqul Islam ARM, Rahman MM. COVID-19 pandemic, socioeconomic crisis and human stress in resource-limited settings: A case from Bangladesh. Heliyon 2020;6. https://doi.org/10.1016/j.heliyon.2020.e04063.

[13] Zhai Y, Du X. Addressing collegiate mental health amid COVID-19 pandemic. Psychiatry Res 2020; 288. https://doi.org/10.1016/j.psychres.2020.113003.

[14] Magson NR, Freeman JYA, Rapee RM, Richardson CE, Oar EL, Fardouly J. Risk and Protective Factors for Prospective Changes in Adolescent Mental Health during the COVID-19 Pandemic. J Youth Adolesc 2021;50:44-57 https://doi.org/10.1007/s10964-020-01332-9.

[15] Neria Y, Sullivan GM. Understanding the mental health effects of indirect exposure to mass trauma through the media. JAMA - J Am Med Assoc 2011;306:1374-5. https://doi.org/10.1001/jama.2011.1358.

[16] Chew QH, Wei KC, Vasoo S, Chua HC, Sim K. Narrative synthesis of psychological and coping responses towards emerging infectious disease outbreaks in the general population: Practical considerations for the COVID-19 pandemic. Singapore Med J 2020;61:350-6. https://doi.org/10.11622/SMEDJ.2020046.

[17] Fardin MA. Covid-19 and anxiety: A review of psychological impacts of infectious disease outbreaks. Arch Clin Infect Dis 2020;15. https://doi.org/10.5812/archcid.102779.

[18] Goodwin GM. Depression and associated physical diseases and symptoms. Dialogues Clin Neurosci 2006; $8: 259-65$. https://doi.org/10.31887/dens.2006.8.2/mgoodwin.

[19] Mamun MA, Griffiths MD. First COVID-19 suicide case in Bangladesh due to fear of COVID-19 and xenophobia: Possible suicide prevention strategies. $\begin{array}{lll}\text { Asian J } & \text { Psychiatr }\end{array}$ https://doi.org/10.1016/j.ajp.2020.102073. 